\title{
Padé approach to top-quark mass effects in gluon fusion amplitudes
}

\author{
Joshua Davies, ${ }^{a}$ Ramona Gröber, ${ }^{b}$ Andreas Maier, ${ }^{c}$ Thomas Rauh ${ }^{* d}$ and Matthias \\ Steinhauser $^{a}$ \\ ${ }^{a}$ Institut f ür Theoretische Teilchenphysik, \\ Karlsruhe Institute of Technology (KIT), \\ Wolfgang-Gaede Straße 1, 76128 Karlsruhe, Germany \\ ${ }^{b}$ Dipartimento di Fisica e Astronomia "G. Galilei”, Università di Padova, and \\ Istituto Nazionale di Fisica Nucleare, Sezione di Padova, \\ I-35131 Padova, Italy \\ ${ }^{c}$ Deutsches Elektronen-Synchrotron, DESY, \\ Platanenallee 6, D-15738 Zeuthen, Germany \\ ${ }^{d}$ Albert Einstein Center for Fundamental Physics, \\ Institute for Theoretical Physics, University of Bern, \\ Sidlerstrasse 5, CH-3012 Bern, Switzerland \\ E-mail: joshua.daviesakit.edu, ramona.groeberapd.infn.it, \\ andreas.martin.maierdesy.de, rauh@itp.unibe.ch, \\ matthias.steinhauserakit.edu
}

\begin{abstract}
Gluon fusion processes like single and double Higgs production exhibit slow convergence and pose severe computational challenges. We show how the top-quark mass dependence of the virtual amplitudes can be reconstructed with a conformal mapping and Padé approximants based on the expansion in the inverse top-quark mass and the non-analytic terms in the expansion around the top threshold. The method is then applied at two- and three-loop order.
\end{abstract}

\footnotetext{
* Speaker.
} 


\section{Introduction}

Following the advent of precision Higgs physics, top-quark mass effects are currently a very active topic, which was also heavily featured at this conference [1]. We show sample diagrams for some of the relevant processes in Figure 1, where the fermion lines correspond to the top quark. These gluon-fusion processes have in common that they are loop-induced and that their perturbative expansions only converge slowly, implying that higher-order corrections are both important and very challenging to compute. Traditionally, the infinite top-quark mass limit has been used beyond leading order to considerably simplify calculations. In many cases, higher powers in the large top-quark mass expansion (LME) were computed to refine predictions and obtain rough estimates for the uncertainties related to the approximation. While the expansion yields reliable results for single on-shell Higgs production, it breaks down in kinematic regimes of $2 \rightarrow 2$ processes where the internal top quarks can be on their mass shell, e.g. at large invariant masses or transverse momenta.

In recent years numerical NLO results have become available [2-6], but these calculations require a high degree of optimization and still pose serious challenges. Therefore, there has also been considerable recent interest in expansion methods which are viable in an extended or complementary part of the phase space [7-10]. Here, we report on a different approach [11] based on the reconstruction of the amplitude from the LME and the expansion around the top-quark threshold $\hat{s}=4 m_{t}^{2}$ with a conformal mapping and Padé approximants [12-14]. This method has the advantage that it is applicable in the entire physical phase space and that calculations are manageable at three-loop order where the other expansion methods are not feasible with the current computational technology. We first review our approach for the simpler case of single-Higgs production [15] and then discuss double-Higgs production [11] and the Higgs-interference contribution to double- $Z$ production [16].

\section{Single-Higgs production}

The amplitude for the production of an off-shell Higgs boson in gluon fusion

$$
\mathscr{A}_{a b}^{\mu v}\left(g\left(p_{1}, \mu, a\right), g\left(p_{2}, v, b\right) \rightarrow H^{(*)}\left(p_{H}\right)\right)=\delta_{a b} T_{F} \frac{y_{t} \hat{s}}{\sqrt{2} m_{t}} \frac{\alpha_{s}}{4 \pi}\left(g^{\mu v}-\frac{p_{1}^{v} p_{2}^{\mu}}{p_{1} \cdot p_{2}}\right) F_{\triangle}(z)
$$
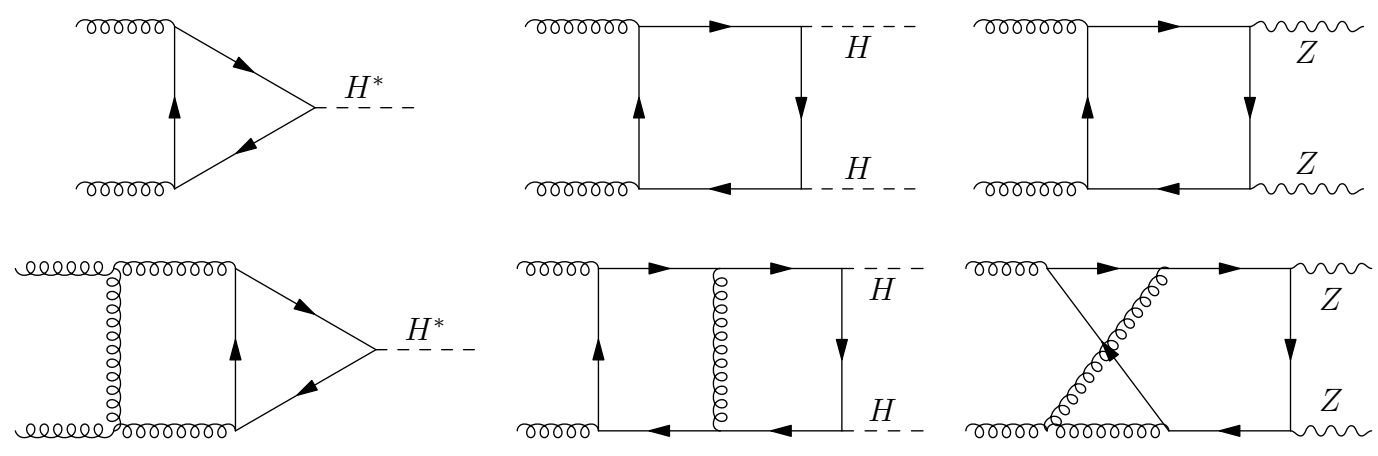

Figure 1: Sample diagrams for gluon fusion processes at LO (top) and NLO (bottom). 


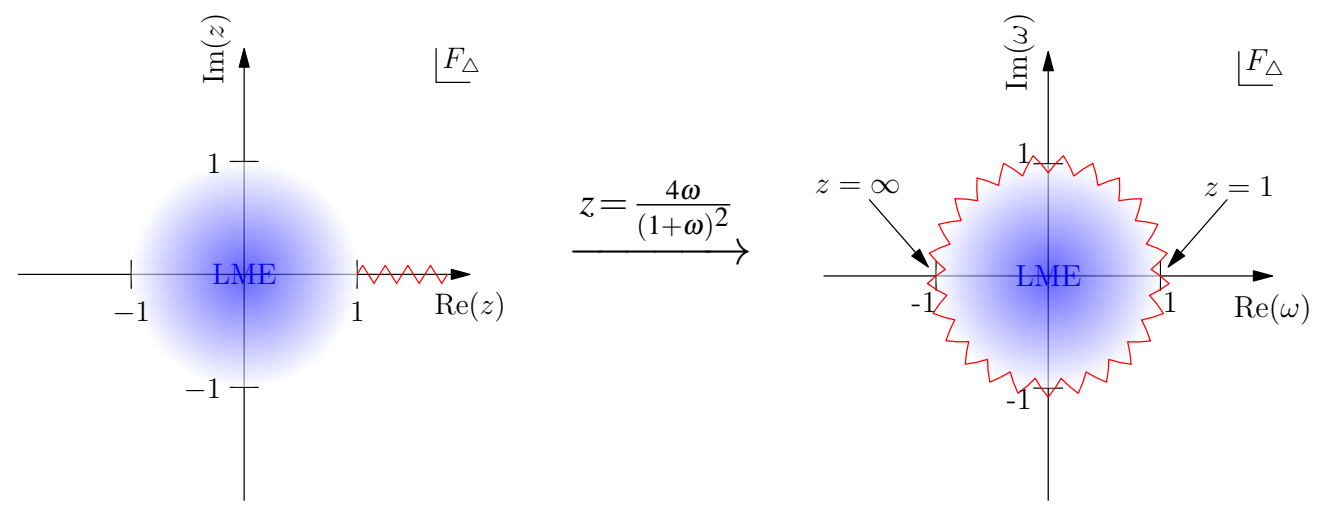

Figure 2: Analytic structure of the triangle form factor in the complex $z$ and $\omega$ planes. The red zigzag curve denotes the $t \bar{t} X$ branch cut and the blue shaded region illustrates the convergence properties of the LME.

only depends on a single form factor $F_{\Delta}(z)$ that is a function of the dimensionless ratio $z=\hat{s} /\left(4 m_{t}^{2}\right)$, where $\hat{s}=\left(p_{1}+p_{2}\right)^{2}$ is the partonic center-of-mass energy. We apply the IR subtractions determined in [17] to define finite form factors $F_{\Delta}^{(1), \text { fin }}$ and $F_{\Delta}^{(2) \text {,fin }}$ at two and three-loop order, respectively. Causality implies that the form factor is an analytic function in the entire complex $z$-plane apart from a branch cut starting at the top-quark threshold $z=1$ as illustrated in Figure 2, which limits the radius of convergence of the LME to unity. Beyond leading order there is also a branch cut from massless intermediate states (e.g. from cutting the gluon loop in the bottom-left diagram in Figure 1) starting at $z=0$ which we will discuss below. We now apply the conformal transformation

$$
z=\frac{4 \omega}{(1+\omega)^{2}}
$$

which maps the complex $z$-plane onto the unit disc $|\omega| \leq 1$ and the branch cut on the perimeter as shown in Figure 2. This implies that the form factor can be approximated with a power series in $\omega(z)$ by determining the coefficients from the condition that the expansion of the ansatz in $z$ must reproduce the LME. A more general approach is to use Padé approximants

$$
[n / m](\omega)=\frac{\sum_{i=0}^{n} a_{i} \omega^{i}}{1+\sum_{j=1}^{m} b_{j} \omega^{j}},
$$

which perform best when the polynomial degrees of the numerator and denominator are similar $n \approx m$. While it is possible to construct Padé approximants with only the LME as an input to determine the coefficients $a_{i}$ and $b_{j}$, the quality of the results deteriorates with increasing loop order. This can be understood from the expansion of the form factor around the top threshold

$$
F_{\Delta}^{(N), \text { fin }} \stackrel{z \rightarrow 1}{=} \sum_{n=0}^{N}\left[\sum_{i=0}^{\infty} A_{i n} \bar{z}^{i}+\sum_{i=3-N}^{\infty} \sum_{j=i \bmod 2}^{N} B_{i j n} \bar{z}^{i / 2} \ln ^{j}(\bar{z})\right] \ln ^{n}(-4 z+i 0),
$$

where $\bar{z}=1-z$ and the $A_{i n}$ and $B_{i j n}$ are coefficients. Again, let us first ignore massless cuts and focus on the contribution with $n=0$. In addition to a power series in $\bar{z}$ there are non-analytic 

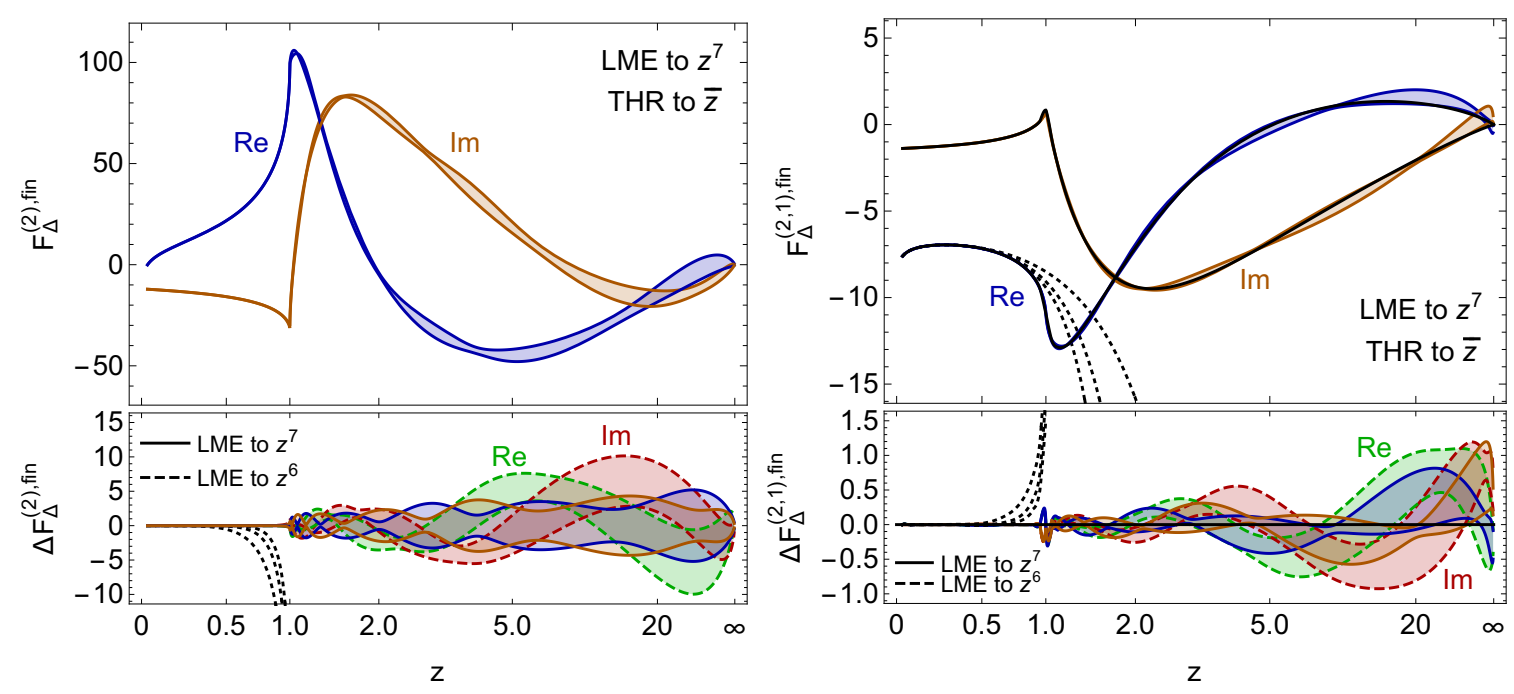

Figure 3: The left plot shows our reconstructed results for the three-loop form factor $F_{\Delta}^{(2), \text { fin }}$ and the right plot compares our approximation of the fermionic contribution $F_{\Delta}^{(2,1) \text {,fin }}$ to the analytic calculation (black) from [24]. The blue and yellow regions correspond to our uncertainty estimate for the real and imaginary part of the form factor. In the lower panel we normalize the result to the central value (left) or the exact result (right) and furthermore show the approximation from [15] where one fewer LME coefficient was known in green and red.

contributions involving square roots and logarithms of $\bar{z}$. The leading power at which these terms appear depends on the loop order due to the $\alpha_{s} / v \sim \alpha_{s} / \bar{z}$ scaling of Coulomb singularities near the threshold. While the roots are tamed by the conformal mapping $\sqrt{\bar{z}}=(1-\omega(z)) /(1+\omega(z))$, which is analytic near $\omega=1$, the logarithms $\ln (\bar{z})=2[\ln (1-\omega(z))-\ln (1+\omega(z))]$ constitute nonanalytic terms near $\omega=1$ which cannot be reproduced by the Padé ansatz (2.3). The appearance of higher powers of these logarithms with increasing loop orders thus explains the worse behaviour of Padé approximants constructed only from the LME.

To improve the reconstruction of the form factor we computed the non-analytic part of the threshold expansion (2.4). For example, for $N=1$ (two loops) we compute $B_{i j 0}$ with $j=1$ for $i=2,4,6$ and $j=0,1$ for $i=3,5{ }^{1}$ This is significantly simpler than the calculation of the analytic terms and sufficient to obtain a good reconstruction of the form factor at least up to three-loop order. The calculation is described in [11] and utilizes the non-relativistic effective field theory methods developed for the computation of the $e^{+} e^{-} \rightarrow t \bar{t} X$ cross section near the top-pair production threshold [18-22]. Our reconstruction takes the form

$$
F_{\triangle}^{(2), \text { fin }}(z) \simeq \frac{[n / m](\omega(z))}{1+a_{R, 0} z}+\frac{[k / l](\omega(z))}{1+a_{R, 1} z} \ln (-4 z+i 0)+s(z)
$$

where the known logarithmic terms have been absorbed into the subtraction function $s(z)$. The remainder is then free of threshold logarithms $\ln (\bar{z})$ up to the computed order in the threshold

\footnotetext{
${ }^{1}$ At two-loop order the terms proportional to $\ln (-4 z+i 0)$ are removed by the IR subtraction.
} 

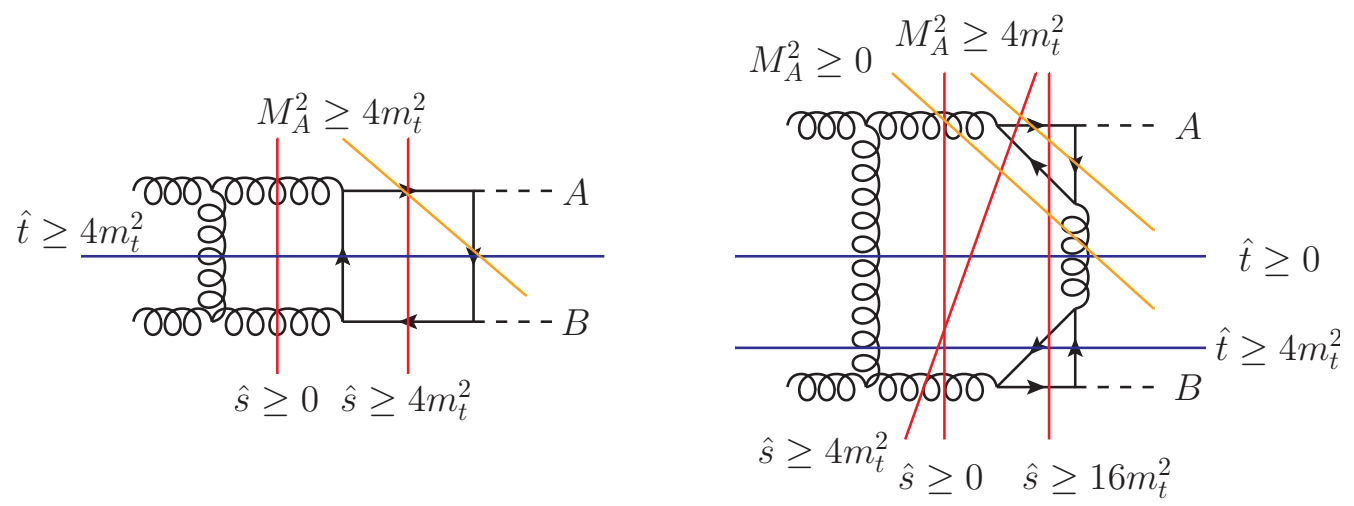

Figure 4: Examples for on-shell cuts in two diagrams contributing to the $g g \rightarrow A B$ process.

expansion and better suited for Padé approximants. Here, we separately reconstruct the coefficient of the logarithm $\ln (-4 z)$ stemming from massless cuts. We use a rescaling with $1+a_{R, i} z$ to enforce the scaling $F_{\Delta} \rightarrow 0$ as $z \rightarrow \infty$ which is a consequence of unitarity. Furthermore, randomly choosing the parameters $a_{R, i}$ in the range $[0.1,10]$ allows us to construct multiple Padé approximants and thus estimate the uncertainty of the results. In [15] we presented our approximants for the three-loop form factor including input from the LME up to the order $z^{6}$ and the threshold expansion up to the order $\bar{z}$.

After the appearance of our work the $z^{7}$ coefficient in the LME has been computed in [23] and the light-fermion contributions have been calculated analytically in [24]. In Figure 3 we update our results from [15] with Padé approximants constructed using the additional LME coefficient and compare the fermionic contribution to the exact calculation. Sample Padé approximants which include the new input are available from [25]. We observe that the uncertainties are tiny below the top threshold and then moderately increase for larger values of $z$ while remaining sufficiently small for phenomenological applications throughout the entire range. The lower panel shows normalized results with and without the $z^{7}$ coefficient and we find good consistency between the two approximations. The new LME coefficient improves the agreement of the light-fermion contribution with the exact results where our earlier result from [15] seems to have slightly underestimated the uncertainty in some parts of the energy range. This demonstrates that the Padé approximants can further be improved by including additional information from the kinematic expansions.

\section{Padé method for $g g \rightarrow A B$ processes}

The method we described in Section 2 can also be applied to the kinematically more complicated amplitudes of $g g \rightarrow A B$ scattering processes. First we decompose the amplitude into a number of form factors which will then be reconstructed. These form factors depend on several kinematic quantities: the invariant masses $M_{A}, M_{B}$ of the final state particles, the Mandelstam variables $\hat{s}, \hat{t}, \hat{u}$ which are subject to the condition

$$
\hat{s}+\hat{t}+\hat{u}=M_{A}^{2}+M_{B}^{2},
$$



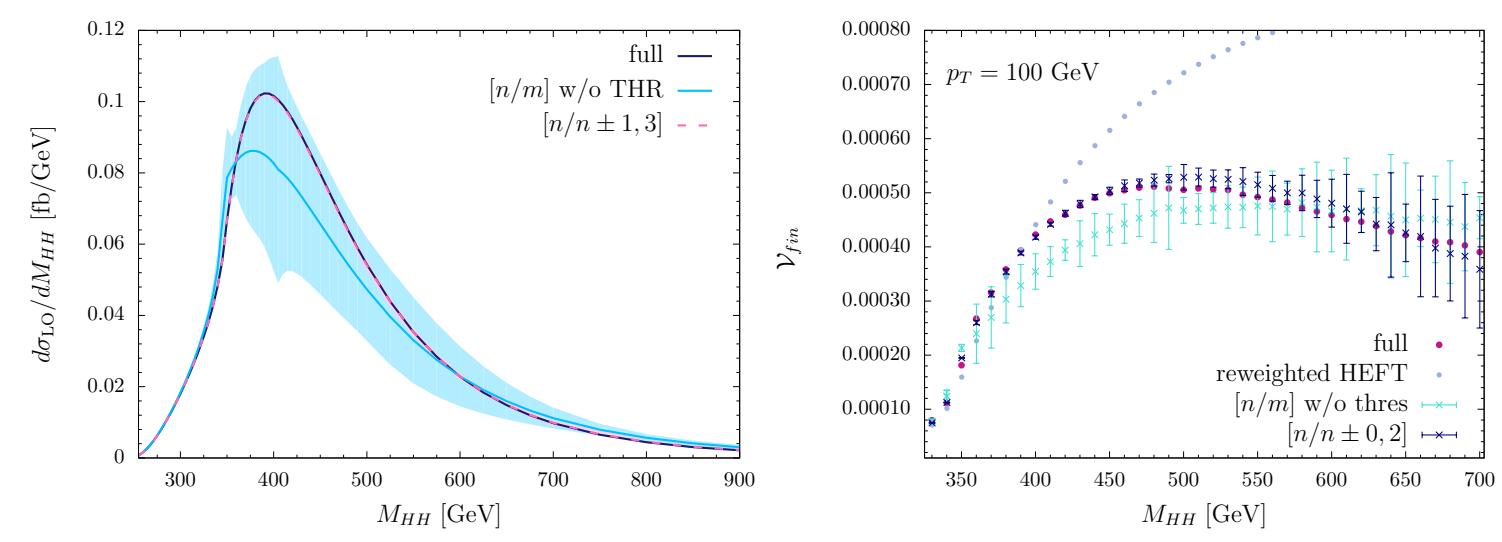

Figure 5: The left plot shows the leading-order invariant mass distribution of Higgs-pair production. The dark blue line is the exact result and the pink-dashed line corresponds to our full reconstruction where the uncertainty is too small to be seen. The light-blue line shows the reconstruction without input from the threshold expansion and the light-blue region corresponds to the uncertainty estimate. On the right we compare the IR-subtracted squared matrix element $\mathscr{V}_{\text {fin }}$ with the numerical calculation from $[2,3]$ (pink points). Our results with and without input from the top threshold are given in dark blue and light blue, respectively.

and the mass $m_{t}$ of the internal top quarks. This implies that the analytic structure of the form factors is much more complicated than for $g g \rightarrow H^{*}$. We show several on-shell cuts in sample diagrams in Figure 4. We divide the form factors into coefficients of logarithms $\ln \left(-\hat{s} / m_{t}^{2}+i 0\right)$, $\ln \left(-M_{A}^{2} / m_{t}^{2}+i 0\right)$, etc. from the massless cuts $\hat{s} \geq 0, M_{A}^{2} \geq 0$, etc. as done for the $\hat{s}$-channel cut in (2.5). These coefficients are then reconstructed separately and we proceed as follows.

First, we specify a phase space point by fixing the kinematic variables $\hat{s}, \hat{t}, \hat{u}, M_{A}^{2}, M_{B}^{2}$ in the physical region. The variable $z$ then encodes the top-quark mass dependence of the amplitude at the given phase space point. The analytic structure is similar to that displayed on the left hand side in Figure 2, but there are also branch cuts at $z \leq \hat{s} / \hat{t}, \hat{s} / \hat{u}$, corresponding to negative values of $m_{t}^{2}$, from the $t$ and $\hat{u}$ channel. These values of $z$ map onto real negative values of $\omega$ inside the unit circle under the conformal transformation (2.2). While the branch cuts cannot be reproduced by the Padé ansatz, the negative values of $m_{t}^{2}$ are obviously unphysical and we observe that the leading-order form factors for $g g \rightarrow H H$ and the Higgs-interference contribution to $g g \rightarrow Z Z$ can be reproduced almost perfectly in spite of them [11,16]. A similar situation has been discussed in [26] where it was shown that the poles in the Padé ansatz (2.3) accumulate in the region of the branch cut and thus approximate the cut. Since the Padé approximants do not need to be evaluated in the vicinity of the $\hat{t}$ and $\hat{u}$ channel thresholds we conclude that the corresponding cuts do not impede the reconstruction of the amplitude with our method. The remaining branch cuts at $\hat{s} \geq 16 m_{t}^{2}$, $M_{A}^{2} \geq 4 m_{t}^{2}$ etc. lie on top of the $t \bar{t} X$ cut with threshold that are significantly larger than $z=1$ and do not pose any problems.

Our approximate result follows by constructing Padé approximants in the variable $\omega(z)$ and evaluating them for the physical value of $m_{t}$. We use random values of the rescaling parameters 

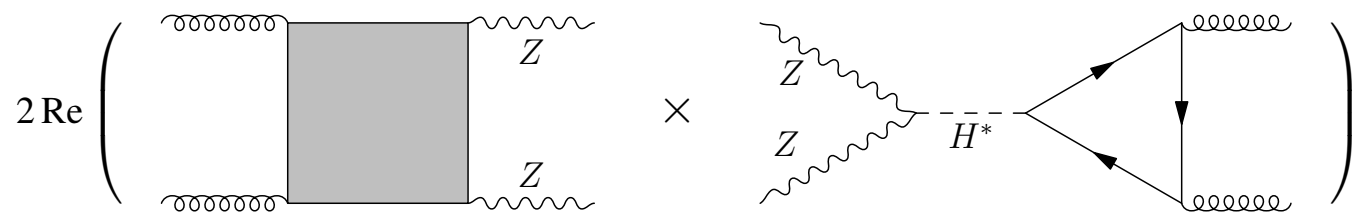

Figure 6: Off-shell Higgs interference contribution to $Z$-pair production in gluon fusion.

$a_{R, i}$ defined as in (2.5) to obtain a total of $100[\mathrm{n} / \mathrm{m}]$ approximants where we apply the condition $|n-m| \leq 3$ since Padé approximants with a similar polynomial degree in the numerator and denominator generally perform best. Furthermore, we exclude approximants with poles close to real positive values of $z$ in the complex plane. The standard deviation of the 100 values is used as an estimate of the uncertainty. These steps need to be performed for every phase space point which typically requires a few CPU seconds.

\subsection{Double-Higgs production}

Double-Higgs production is the best studied case of top-quark mass effects in gluon-fusion processes with two-particle final states where the first numerical two-loop calculations [2,3] appeared. This allows a detailed comparison of our approach which is shown in Figure 5. We constructed Padé approximants from the LME up to the order $z^{4}[27,28]$ and the threshold expansion up to the order $\bar{z}^{5 / 2}$ (LO) and $\bar{z}^{2}$ (NLO) [11]. This provides an almost perfect reconstruction at LO with tiny uncertainties. The comparison with the approximation without input from the threshold expansion demonstrates the importance of that additional information for the method. At NLO we find that the reconstructed result matches the numerical calculation very well, albeit with bigger errors that increase towards larger invariant masses. Larger uncertainties are expected because most of the complications discussed above, i.e. massless cuts and threshold logarithms, first appear at two-loop order. In particular we find that the coefficient of the logarithm $\ln \left(-\hat{s} / m_{t}^{2}+i 0\right)$ from the massless $\hat{s}$-channel cut has a larger relative uncertainty than the non-logarithmic term. Still, the uncertainties are small enough for phenomenological applications and can be further reduced with more input from the LME and the threshold expansion.

\subsection{Higgs-interference contribution to $Z$-pair production}

Measuring the process $g g \rightarrow H \rightarrow Z Z(\rightarrow 4 l)$ on and off the Higgs-boson peak provides an indirect way to measure the Higgs-boson width $\Gamma_{H} \sim 4 \mathrm{MeV}$ [29-31], which is not directly accessible at the LHC or the planned next generation of collider experiments due to insufficient energy resolution. A numerically important but computationally challenging contribution to the off-shell regime is the interference contribution shown in Figure 6 where the top-quark contribution to the $g g \rightarrow Z Z$ amplitude dominates the region of large invariant mass $M_{Z Z}$. The top-quark contribution has been computed at two-loop order within the LME $[32,33]$ and Padé approximants based on the LME were studied in [33]. Only two form factors contribute to the Higgs-interference contribution and we show our approximate results based on the LME to $z^{6}$ and the threshold expansion up to at least $\bar{z}^{4}[16]$ in Figure 7. Again we obtain an almost perfect reconstruction at LO. At NLO we get a very good prediction for the numerically dominant axial-vector form factor $\left|\widetilde{F}_{A A}^{(2)}\right\rangle$ in the entire 

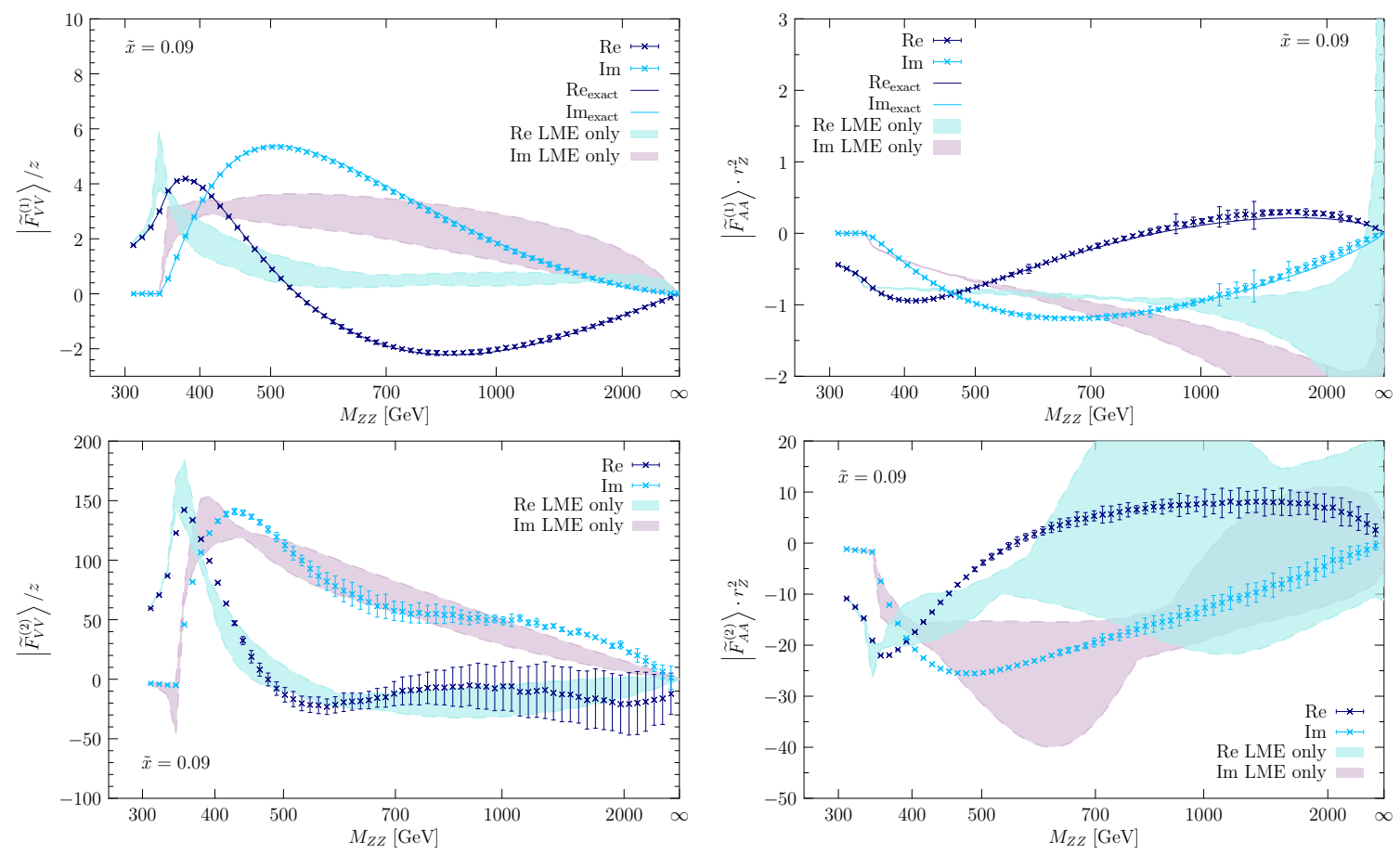

Figure 7: The LO (top) and NLO (bottom) form factors $\left|\widetilde{F}_{V V}^{(i)}\right\rangle$ (left) and $\left|\widetilde{F}_{A A}^{(i)}\right\rangle$ are shown as a function of the invariant mass of the $Z$-boson pair at a fixed value of $\tilde{x}=\left(p_{T}^{2}+m_{Z}^{2}\right) / M_{Z Z}^{2}=0.09$. The real and imaginary parts of the Padé approximants are shown in dark blue and light blue and the solid lines correspond to the exact result at LO. The shaded regions show an approximation constructed from the LME only, following the approach of [33].

phase space. The errors are larger and the convergence is slower for the vector form factor $\left|\widetilde{F}_{V V}^{(2)}\right\rangle$, because the contribution proportional to $\ln \left(-\hat{s} / m_{t}^{2}+i 0\right)$ is larger relative to the non-logarithmic term and behaves worse as already mentioned for the case of Higgs-pair production. However, the discussion in [16] demonstrates that the interference amplitude can be described reliably due to the smallness of the vector form factor.

\section{Conclusions}

We have described our method for the reconstruction of top-quark mass effects in gluon fusion processes with a conformal mapping and Padé approximants based on kinematic expansions. The reliability of the approach has been demonstrated with examples at two and three-loop order. While more numerical calculations have become available at two-loop order [1-6], our method is the only approach beyond the LME that is feasible at three loops with the current computational technology and can significantly improve the quality of predictions at this order [34].

\section{References}

[1] M. Kerner, Top mass effects in HJ and HH production, 
$\mathrm{X}$. Zhao, Top-quark effects in diphoton production through gluon fusion at NLO in QCD,

B. Agarwal, On gg $\rightarrow \mathrm{ZZ}$ production at two loops with full top mass dependence,

L. Chen, Photon pair production in gluon fusion: Top quark threshold effects,

M. Steinhauser, Higher order corrections to Higgs boson pair production, contributions to these proceedings.

[2] S. Borowka et al., Higgs Boson Pair Production in Gluon Fusion at Next-to-Leading Order with Full Top-Quark Mass Dependence, Phys. Rev. Lett. 117 (2016) no.1, 012001 Erratum: [Phys. Rev. Lett. 117 (2016) no.7, 079901] [arXiv:1604.06447 [hep-ph]].

[3] S. Borowka at al., Full top quark mass dependence in Higgs boson pair production at NLO, JHEP 1610 (2016) 107 [arXiv:1608.04798 [hep-ph]].

[4] S. P. Jones, M. Kerner and G. Luisoni, Next-to-Leading-Order QCD Corrections to Higgs Boson Plus Jet Production with Full Top-Quark Mass Dependence, Phys. Rev. Lett. 120 (2018) no.16, 162001 [arXiv:1802.00349 [hep-ph]].

[5] J. Baglio at al., Gluon fusion into Higgs pairs at NLO QCD and the top mass scheme, Eur. Phys. J. C 79 (2019) no.6, 459 [arXiv:1811.05692 [hep-ph]].

[6] L. Chen et al., Photon pair production in gluon fusion: Top quark effects at NLO with threshold matching, arXiv:1911.09314 [hep-ph].

[7] R. Bonciani, G. Degrassi, P. P. Giardino and R. Gröber, Analytical Method for Next-to-Leading-Order QCD Corrections to Double-Higgs Production, Phys. Rev. Lett. 121 (2018) no.16, 162003 [arXiv:1806.11564 [hep-ph]].

[8] J. Davies, G. Mishima, M. Steinhauser and D. Wellmann, Double-Higgs boson production in the high-energy limit: planar master integrals, JHEP 1803 (2018) 048 [arXiv:1801.09696 [hep-ph]].

[9] J. Davies, G. Mishima, M. Steinhauser and D. Wellmann, Double Higgs boson production at NLO in the high-energy limit: complete analytic results, JHEP 1901 (2019) 176 [arXiv:1811.05489 [hep-ph]].

[10] X. Xu and L. L. Yang, Towards a new approximation for pair-production and associated-production of the Higgs boson, JHEP 1901 (2019) 211 [arXiv:1810.12002 [hep-ph]].

[11] R. Gröber, A. Maier and T. Rauh, Reconstruction of top-quark mass effects in Higgs pair production and other gluon-fusion processes, JHEP 1803 (2018) 020 [arXiv:1709.07799 [hep-ph]].

[12] P. A. Baikov and D. J. Broadhurst, Three loop QED vacuum polarization and the four loop muon anomalous magnetic moment, In *Pisa 1995, New computing techniques in physics research* 167-172 [hep-ph/9504398].

[13] D. J. Broadhurst, J. Fleischer and O. V. Tarasov, Two loop two point functions with masses: Asymptotic expansions and Taylor series, in any dimension, Z. Phys. C 60 (1993) 287 [hep-ph/9304303].

[14] J. Fleischer and O. V. Tarasov, Calculation of Feynman diagrams from their small momentum expansion, Z. Phys. C 64 (1994) 413 [hep-ph/9403230].

[15] J. Davies, R. Gröber, A. Maier, T. Rauh and M. Steinhauser, Top quark mass dependence of the Higgs boson-gluon form factor at three loops, Phys. Rev. D 100 (2019) no.3, 034017 [arXiv:1906.00982 [hep-ph]].

[16] R. Gröber, A. Maier and T. Rauh, Top quark mass effects in gg $\rightarrow$ ZZ at two loops and off-shell Higgs interference, arXiv:1908.04061 [hep-ph]. 
[17] S. Catani, The Singular behavior of QCD amplitudes at two loop order, Phys. Lett. B 427 (1998) 161 [hep-ph/9802439].

[18] M. Beneke et al., Next-to-Next-to-Next-to-Leading Order QCD Prediction for the Top Antitop S-Wave Pair Production Cross Section Near Threshold in $e^{+} e^{-}$Annihilation, Phys. Rev. Lett. 115 (2015) no.19, 192001 [arXiv:1506.06864 [hep-ph]].

[19] M. Beneke, J. Piclum and T. Rauh, P-wave contribution to third-order top-quark pair production near threshold, Nucl. Phys. B 880 (2014) 414 [arXiv:1312.4792 [hep-ph]].

[20] M. Beneke, A. Maier, J. Piclum and T. Rauh, Higgs effects in top anti-top production near threshold in $e^{+} e^{-}$annihilation, Nucl. Phys. B 899 (2015) 180 [arXiv:1506.06865 [hep-ph]].

[21] M. Beneke, Y. Kiyo, A. Maier and J. Piclum, Near-threshold production of heavy quarks with QQbar_threshold, Comput. Phys. Commun. 209 (2016) 96 [arXiv:1605.03010 [hep-ph]].

[22] M. Beneke, A. Maier, T. Rauh and P. Ruiz-Femenia, Non-resonant and electroweak NNLO correction to the $e^{+} e^{-}$top anti-top threshold, JHEP 1802 (2018) 125 [arXiv:1711.10429 [hep-ph]].

[23] J. Davies and M. Steinhauser, Three-loop form factors for Higgs boson pair production in the large top mass limit, JHEP 1910 (2019) 166 [arXiv:1909.01361 [hep-ph]].

[24] R. V. Harlander, M. Prausa and J. Usovitsch, The light-fermion contribution to the exact Higgs-gluon form factor in QCD, JHEP 1910 (2019) 148 [arXiv:1907.06957 [hep-ph]].

[25] https://www.ttp.kit.edu/preprints/2019/ttp19-044/

[26] P. Masjuan and S. Peris, Pade Theory applied to the vacuum polarization of a heavy quark, Phys. Lett. B 686 (2010) 307 [arXiv:0903.0294 [hep-ph]].

[27] J. Grigo, J. Hoff and M. Steinhauser, Higgs boson pair production: top quark mass effects at NLO and NNLO, Nucl. Phys. B 900 (2015) 412 [arXiv:1508.00909 [hep-ph]].

[28] G. Degrassi, P. P. Giardino and R. Gröber, On the two-loop virtual QCD corrections to Higgs boson pair production in the Standard Model, Eur. Phys. J. C 76 (2016) no.7, 411 [arXiv:1603.00385 [hep-ph]].

[29] N. Kauer and G. Passarino, Inadequacy of zero-width approximation for a light Higgs boson signal, JHEP 1208 (2012) 116 [arXiv:1206.4803 [hep-ph]].

[30] F. Caola and K. Melnikov, Constraining the Higgs boson width with ZZ production at the LHC, Phys. Rev. D 88 (2013) 054024 [arXiv:1307.4935 [hep-ph]].

[31] J. M. Campbell, R. K. Ellis and C. Williams, Bounding the Higgs Width at the LHC Using Full Analytic Results for gg-> $e^{-} e^{+} \mu^{-} \mu^{+}$, JHEP 1404 (2014) 060 [arXiv:1311.3589 [hep-ph]].

[32] F. Caola et al., QCD corrections to vector boson pair production in gluon fusion including interference effects with off-shell Higgs at the LHC, JHEP 1607 (2016) 087 [arXiv:1605.04610 [hep-ph]].

[33] J. M. Campbell, R. K. Ellis, M. Czakon and S. Kirchner, Two loop correction to interference in $g g \rightarrow Z Z$, JHEP 1608 (2016) 011 [arXiv:1605.01380 [hep-ph]].

[34] J. Davies, R. Gröber, A. Maier, T. Rauh and M. Steinhauser, in preparation. 